\title{
Utilization of Telehealth Services During the COVID-19 Pandemic
}

\author{
Paige Hoyer, MD; Renat Ahatov, BSA; Lindy Ross, MD
}

\section{PRACTICE POINTS}

- Telehealth can increase access to dermatologic care for both inpatient hospital consultations and outpatient clinic visits, especially in areas lacking dermatologists.

- With the current iPLEDGE accommodations for coronavirus disease 19, we have been able to treat patients who live 3 hours away and cannot travel for monthly isotretinoin visits.

- Telehealth allows our providers to better triage benign vs potentially malignant conditions to schedule patients in a more appropriate time frame.

I 2017, lawmakers and insurers in the state of Texas approved the use of telehealth services in times of crisis. ${ }^{1}$ During the coronavirus disease 2019 (COVID-19) pandemic, our clinic has used telemedicine to provide remote care to dermatology patients. We posit that the quick introduction and implementation of telemedicine during this time of need will change the way we practice dermatology in the future.

At the University of Texas Medical Branch in Galveston, Texas, we primarily have used 2 forms of telemedicine during the COVID-19 pandemic: live face-to-face video communication (our institution primarily uses FaceTime), and a combination of telephone calls with store-andforward images. All dermatology services at our institution were converted to telemedicine visits, and in-person office visits were only done if deemed necessary after triage by telemedicine in April and May 2020. This strategy removed the necessity for patients to leave their homes for their appointments, which not only saved them travel costs and time but also reduced the potential spread of COVID-19. Since this time, the clinic has reopened for in-person visits; however, patients still have the option to schedule a telehealth appointment if they prefer. Many patients still select the telehealth option for the above reasons.

Although routine skin checks were not always possible by video and/or store-and-forward images, telemedicine worked very well for follow-up visits, especially isotretinoin follow-ups. During the COVID-19 outbreak, iPLEDGE (https://www.ipledgeprogram.com /iPledgeUI/home.u) rapidly adapted to the use of telemedicine and even began to allow home pregnancy tests to be entered into the iPLEDGE system by health care providers. Isotretinoin follow-ups are especially useful for patients who do not require laboratory monitoring at the visit. Patients are easily evaluated, screened for side effects, and continued on their treatment if no concerns are found during the telemedicine visit. Patients who require laboratory monitoring are still able to schedule tests at our clinics or at free-standing laboratories near their homes without having an in-office dermatology appointment. At-home pregnancy tests are still being utilized as an option for patients electing for telehealth follow-ups. This strategy is both health conscious by protecting the patient from exposure to COVID-19 at a testing center and cost-effective, especially for our uninsured patients, while still meeting the safety check for iPLEDGE.

Additionally, we utilized store-and-forward telemedicine for hospital consultations. If the patient's condition can easily be diagnosed by viewing unedited clinical

From the University of Texas Medical Branch, Galveston. Drs. Hoyer and Ross are from the Department of Dermatology, and Mr. Ahatov is from the School of Medicine.

The authors report no conflict of interest.

Correspondence: Paige Hoyer, MD, University of Texas Medical Branch, Department of Dermatology, 301 University Blvd, 4.112, McCullough Bldg, Galveston, TX 77555-1327 (pehoyer@utmb.edu).

doi:10.12788/cutis.0034 
images remotely, the clinician can further decrease the risk of COVID-19 spread and exposure by providing the consultation and treatment recommendations by telephone. In cases in which a diagnosis could not be made by reviewing clinical photographs remotely, an in-person visit would be done. We continue to use this strategy for our confirmed COVID-positive hospital consultations to help protect our faculty and residents and decrease the use of personal protective equipment. We propose this model could be instituted for patients admitted to hospitals without access to dermatology consultations. Store-and-forward photographs of worrisome lesions and rashes also can be used to triage visits. For example, a patient with a new-onset keratoacanthoma and a history of nonmelanoma skin cancer contacted our clinic during the pandemic and sent store-and-forward images for review. The patient was triaged by a telemedicine visit and was then brought into the clinic for biopsy based on his clinical photographs and history. Patients also have requested prescriptions for bimatoprost and tretinoin via telehealth, a service that many medical spas and online telehealth companies provide already but was not offered at our practice until now.

Telemedicine also has potentially helped decrease the number of patients going to urgent care clinics for dermatology-related issues. Additionally, we have utilized one provider per day to be the "on-call" dermatologist who would be doing telemedicine appointments for patients with new-onset conditions. This strategy not only minimized possible patient exposure to COVID-19 but also helped preserve resources at urgent care clinics and emergency departments, which currently are inundated with patients. Since we have reopened for in-person visits, we have been unable to sustain an on-call dermatologist for telemedicine but may re-employ this strategy in the future.

The unique experience of practicing medicine during a pandemic has and will affect the way we practice moving forward. The way telemedicine has been quickly and easily implemented by the health care community during the COVID-19 pandemic has taught our dermatologists the value of this method of health care delivery. We will likely continue to use telemedicine after the pandemic has been contained. Telemedicine has the potential to expand access to care to rural and underserved areas, hospitals without on-call dermatologists, and homebound patients. We also may be better able to provide isotretinoin to our patients who have deferred treatment due to difficulty with transportation to the monthly visits. Store-andforward images could help patients referred to dermatology avoid long wait times for obvious skin cancers that would benefit from early treatment. Telemedicine visits also could potentially improve attendance for patients who forget about their appointment by calling them after they miss their scheduled appointment time and complete a telehealth encounter on the same day instead, which could help recover costs of no-show appointments for clinics.

It is still unclear how private insurance companies will adapt to the new use of telemedicine, but we hope they follow the lead of Medicare, which released a statement on March 6, 2020, supporting the implementation of telehealth services. ${ }^{2}$ Although Medicare has made adjustments to allow for equal reimbursement for telehealth appointments, private insurance companies still vary greatly. Many practices are struggling and some remained open despite shelter-in-place orders, but we propose telemedicine may be a safer alternative for patients and providers during the current health crisis that would keep billable services in place. It is still uncertain whether the laws enacted to make telemedicine accessible during this time will hold after COVID-19 is contained, but we are hopeful that living through the pandemic will bring some positive benefit to our practice and the patients we serve.

\section{REFERENCES}

1. Texas laws and regulations relating to telemedicine. Texas Medical Association website. https://www.texmed.org/Template. aspx?id=47554. Updated March 19, 2020. Accessed July 14, 2020.

2. Centers for Medicare \& Medicaid Services. President Trump expands telehealth benefits for Medicare beneficiaries during COVID 19 outbreak. https://www.cms.gov/newsroom/press-releases/president-trump -expands-telehealth-benefits-medicare-beneficiaries-during-covid -19-outbreak. Published March 17, 2020. Accessed July 14, 2020. 